\title{
Evaluation of Changes in Perceived Intensity and Threshold of Moisture Sensation of Clothes Associated with Skin Moisture
}

\author{
Shanyi You ${ }^{(凶)}$, Mai Shibahara, and Katsunari Sato \\ Nara Women's University, Kitauoyahigashicho, Nara, Japan \\ taz_yuu@cc.nara-wu.ac.jp
}

\begin{abstract}
Moisture sensation is an important determinant of clothing comfort. Conventional studies have attempted to elucidate the mechanism behind moisture sensation by using wet sample cloths that vary by water content. However, these studies did not consider the impact of the moisture levels of the skin that makes contact with the samples. In this study, we investigated changes in skin moisture sensation in terms of perceived strength and detection thresholds, based on contact with sample cloths, given various skin moisture conditions. In the first experiment, participants reported their perceived moisture levels for sample cloths that varied in water content and temperature, after making forearm contact with each sample cloth. The result showed that participants felt small amounts of moisture when skin moisture was increased. In the second experiment, participants' detection thresholds were evaluated using the staircase method, based on forearm-sample contact. The results showed that skin moisture did not affect the threshold of moisture sensation.
\end{abstract}

Keywords: Moisture sensation $\cdot$ Skin moisture $\cdot$ Perceived intensity $\cdot$ Threshold

\section{Introduction}

Clothing comfort is a complex issue. It can be influenced by several factors, including human physiology, human psychology, fabric characteristics, and climate [1]. For clothing that makes skin contact, moisture sensation is also an important factor in clothing comfort. However, the mechanism behind the perception of moisture sensation is not fully understood.

Conventional studies of moisture sensation have shown that the major factor affecting moisture sensation is changes in temperature or heat flow [2]. It has previously been found that moisture sensation varied for different parts of the skin [3]. Perceived humidity has been found to vary according to static and dynamic touch but to be independent of changes in pressure. Specifically, in the case of dynamic touch, participants rated stimuli that were dry and cold as being drier than similar stimuli with relatively higher temperatures [4]. Conversely, in the case of static touch, contact with a dry, cold cloth has been shown to lead to temperature changes in the skin that mirror 
those that result from contact with a damp cloth. This represents a case of the perception of illusory moisture [5].

These and other conventional studies focused on the moisture level of the object in question (e.g., fabric) but did not consider effects attributable to the moisture level of the skin. The sensation of moisture in clothing is caused by the contact between the fabric and the skin. If the skin is wet to any degree, heat transfer between the fabric and the skin changes accordingly. Since heat transfer is an important factor in moisture sensation, the moisture level of the skin could also affect sensation.

In this study, we focused on the moisture level of the skin and evaluated changes in the moisture sensation by controlling the water content and temperature of the wet cloth. The change in the threshold of moisture sensation was measured as well. We expect that this study will contribute to the understanding of moisture perception and to improving the comfort of clothing that comes into direct contact with the skin.

\section{Experiment 1: Perceived Intensity of Moisture Sensation}

Perceived intensity of moisture sensation is the degree to which a person can feel moisture when the skin is in contact with a physical entity (e.g., a fabric). In order to confirm changes in the perceived intensity of moisture sensation, based on different skin moisture conditions, we had participants evaluate the strength of moisture sensation when their forearms touched sample cloths with varying temperatures and moisture levels.

\subsection{Experimental Materials}

The size of the sample cloths (cotton broad) was $6.5 \mathrm{~cm}^{2}$. Each cloth was affixed to a $4 \mathrm{~cm}^{2}$ Peltier element, for the purpose of temperature adjustment. A hotplate (Nissin Kogyo Co., Ltd., NHP-M30N) set at $33{ }^{\circ} \mathrm{C}$ was prepared for adjusting the skin temperature of the arm before it came into contact with the sample cloth. Skin moisture levels were measured by using a triplesense (MORITEX Co., Ltd., TR-3).

\subsection{Experimental Conditions}

The participants were 20 female university students. This experiment was approved by the Ethics Review Committee of Nara Women's University. The participants were informed of the relevant experimental procedure, and the experiment was conducted with each participant's consent. The test area in the experiment was the forearm segment of the dominant hand, $5 \mathrm{~cm}$ proximal to the wrist joint. The room temperature and environmental humidity were kept at $23{ }^{\circ} \mathrm{C} \pm 0.5{ }^{\circ} \mathrm{C}$ and $50 \pm 2 \% \mathrm{RH}$, respectively, throughout the experiment.

For each trial, the skin temperature was adjusted to one of two levels: $27{ }^{\circ} \mathrm{C}$ (below normal skin temperature) and $39{ }^{\circ} \mathrm{C}$ (above normal skin temperature). The moisture content of the sample cloth was adjusted to one of three levels: dry, low moisture, and high moisture. Using a dropper, the wet sample clothes were prepared with $100 \mu \mathrm{l}$ and $300 \mu \mathrm{l}$ of water for the low and high moisture conditions, respectively. During the experiment, skin moisture was adjusted using a wet towel. 
Moisture sensation was evaluated using a four-point Likert scale, ranging from 0-3, as shown in Fig. 1. We selected the four-point scale, as previous experiments [5] have shown that participants can easily and precisely express their sensations of moisture, using this a scale of this length. We analyzed these values on an interval scale [6].

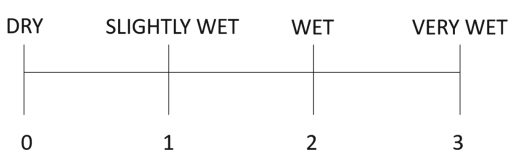

Fig. 1. Moisture sensation rating scale.

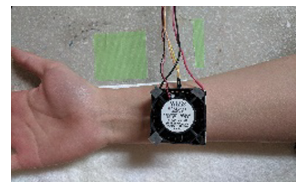

Fig. 2. Forearm experiment.

\subsection{Experimental Procedure}

Prior to the experiment, participants were asked to touch the dry and high-moisture sample cloths in order to ensure they had knowledge of the two ends ( 0 and 3 ) of the scale. Then, the initial skin moisture of participants' forearm was measured by the triplesense.

The participants closed their eyes during the experiment, so as not to see the sample cloth. First, the skin temperature was adjusted with the hot plate for $1 \mathrm{~min}$. Next, the experimenter guided each participant's dominant hand to their side and brought the sample cloth into contact with the forearm for $4 \mathrm{~s}$ (Fig. 2). The forearm was then removed from the sample cloth and moisture sensation was evaluated. Afterwards, the arm was returned to the hot plate to restore each participant's skin temperature. In the above procedure, six conditions with different levels of water content and different temperatures were evaluated in random order for each participant. Each condition was repeated once.

Thereafter, a wet towel was placed on the arms of each participant for $5 \mathrm{~min}$ to adjust skin moisture. After that, the Triplesense was used to confirm the projected increase in skin moisture. This procedure was repeated six times (varied according to condition) to evaluate outcomes in each of the six conditions.

\subsection{Results}

Skin moisture levels before and after adjustment are shown in Fig. 3. We confirmed that skin moisture content was successfully increased for each participant, following adjustment.

Averages of the results of the four-step evaluations were calculated, based on moisture sensation ratings given before and after skin moisture adjustment, in each condition. Summaries of moisture sensation ratings are shown in Fig. 4.

It can be seen from Fig. 4 that, when the wet towel was not used (i.e., skin moisture was low), the moisture sensation rating was higher than when the wet towel was used (i.e., skin moisture was high). This indicates that, when the skin moisture was high, the moisture was perceived very little. 


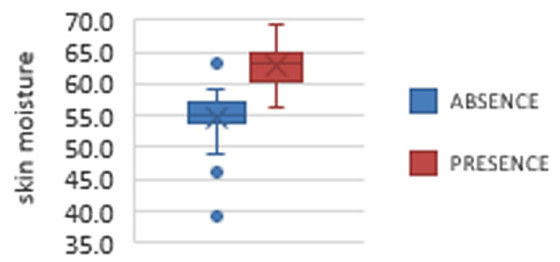

Fig. 3. The skin moisture content before and after the adjustment in Experiment 1.

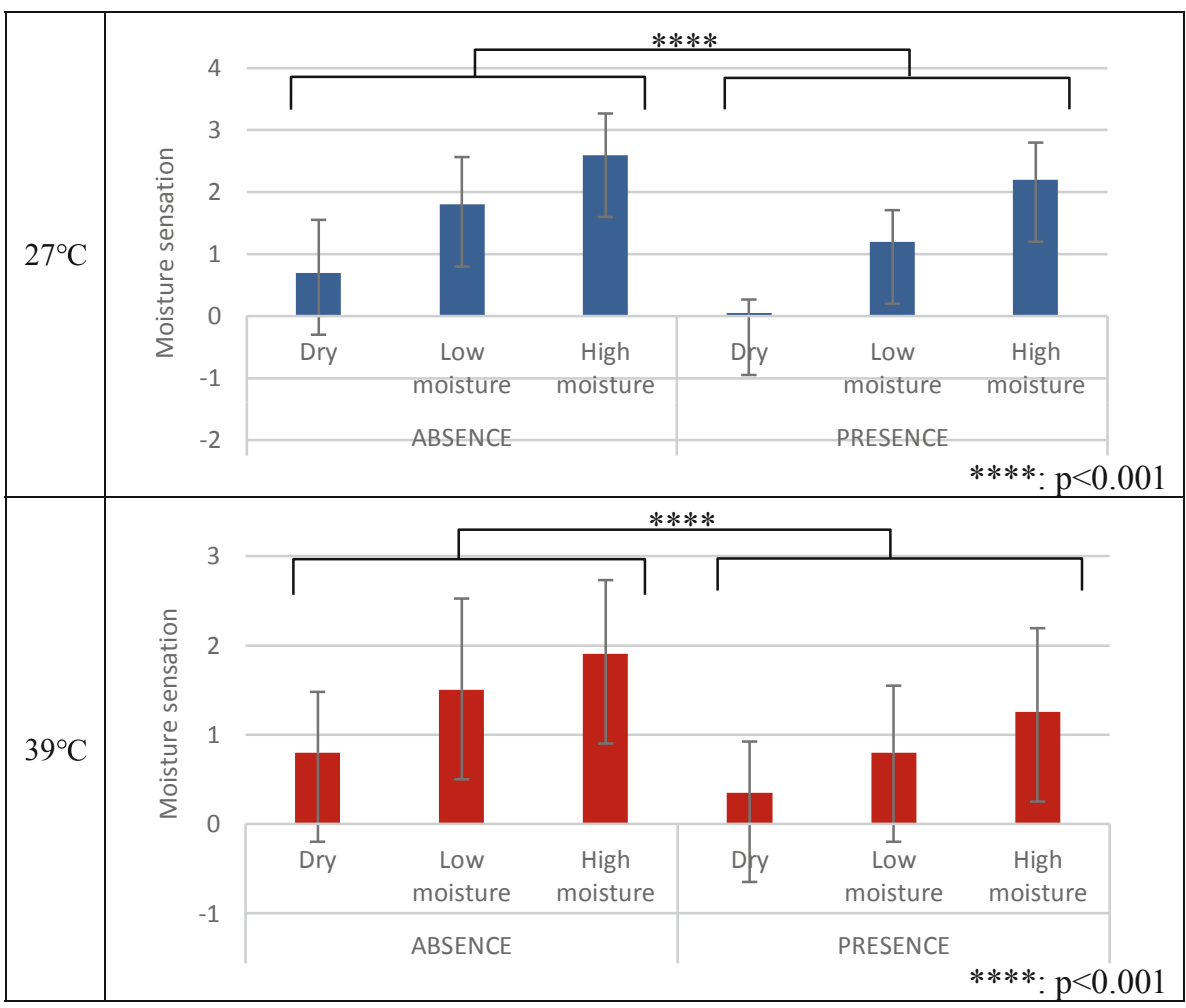

Fig. 4. Moisture sensation at $27{ }^{\circ} \mathrm{C}$ and $39{ }^{\circ} \mathrm{C}$. The horizontal axis shows the moisture content of the sample cloth and the skin. The vertical axis shows the moisture sensation ratings. The errors bars represent standard deviations.

A 2 (temperature) $\times 3$ (cloth moisture) $\times 2$ (skin moisture) ANOVA, at $\alpha=0.05$, revealed a significant difference according to skin moisture $(F(1,19)=26.11$, $p<0.001)$. In addition, the temperature $\times$ cloth moisture interaction was significant. Multiple comparisons showed no significant simple main effects in the dry and lowmoisture conditions, but showed a significant effect in the high-moisture condition $(F$ $(1,57)=17.17, p<0.001)$. Furthermore, there were significant differences within the 
$27{ }^{\circ} \mathrm{C}(F(2,76)=84.77, p<0.001)$ and $39{ }^{\circ} \mathrm{C}(F(2,76)=20.66, p<0.001)$ temperature conditions. Further, multiple comparisons with post-hoc tests, using Ryan's method, showed that there were significant differences among three cloth moisture conditions $(p<0.001)$ in both temperature conditions.

Figure 5 depicts the relationship between recorded levels of skin moisture and participant-rated moisture sensation. No significant correlation was found between these measures $\left(27^{\circ} \mathrm{C}: \mathrm{R}(\right.$ Dry $)=-0.60, \mathrm{R}($ Low $)=0.11, \mathrm{R}($ High $)=-0.33,39^{\circ} \mathrm{C}: \mathrm{R}$ $($ Dry $)=-0.28, \mathrm{R}($ Low $)=-0.08, \mathrm{R}($ High $)=0.09)$.

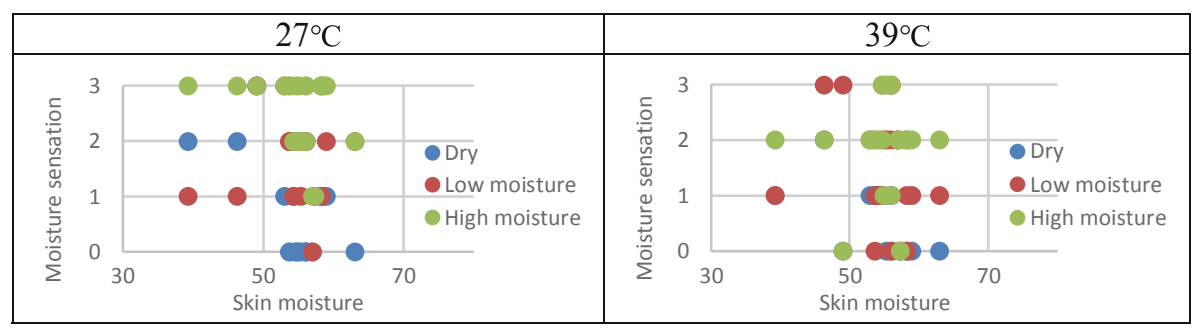

Fig. 5. The relationship between skin moisture and moisture sensation at $27{ }^{\circ} \mathrm{C}$ and $39{ }^{\circ} \mathrm{C}$. The horizontal axis shows skin moisture, and the vertical axis shows moisture sensation ratings. The different dot colors represent the cloth moisture conditions. (Color figure online)

\subsection{Discussion}

Conventional studies of moisture sensation have focused on the moisture levels of objects such as a cloth. These studies have found that higher levels of moisture are associated with higher levels of heat transfer, making it easier to feel moistness [3, 5]. Similarly, it has been shown that the heat transfer increased when skin moisture was higher. Conventional studies [7] have shown that the amount of heat transfer was dependent on the thermal contact coefficient $h$ :

$$
h=(k \rho c)^{1 / 2}
$$

where $k$ is thermal conductivity, $c$ is specific heat capacity and $\rho$ is density. Moisture had a higher thermal contact coefficient than skin, when the movement of heat was larger. However, the current study (Fig. 4) showed that moisture sensation ratings decreased when skin moisture increased. This trend depended not on absolute skin moisture content but on relative content within individuals (Fig. 5).

Comprehensive judgments of moisture levels have been found to vary, based on friction and softness, in addition to temperature. Conventional studies have shown that contact with a cold object, with a high surface friction level, made people perceive the object as soft and moist [5, 8, 9]. In this experiment, skin moisture increased after the wet towel was applied. It is possible that, as the skin became softer, the sample cloth increased in perceived, relative hardness. It is presumed that this would have impaired individuals' ability to feel moistness. Additionally, when skin moisture levels 
increased, the number of active mechanoreceptors might have changed, correspondingly. Further research is required to test this assumption. In addition, skin sensations before and after contact with the sample cloth could be affected. It is possible that the amount of water on the skin's surface changed slightly when the moist stimulus touched the skin. If judgments of moisture levels are based on the difference in sensation between pre- and post-contact, it should be easier to perceive the change when the skin is dry, initially.

Changes in clothes' perceived moisture levels depended on the skin's moisture content. This is an important phenomenon not only for the design and development of comfortable clothing but also for psychophysical research. For example, since the skin temperature affects thermal and vibro-tactile stimulation, we adjusted the skin temperature before the experiment $[10,11]$. Our results indicate that, during research measuring perceived moisture levels, it should be confirmed that the moisture content of the skin does not change during the experiment.

\section{Experiment 2: The Threshold of the Moisture Sensation}

The threshold of moisture sensation is the minimum water content required to illicit the feeling of moisture on the skin. We sought to confirm changes in the threshold of moisture sensation on the skin, based on different skin moisture conditions. As such we measured changes in sensation, based on cloths of varying moisture content coming into contact with skin with varying surface moisture levels.

\subsection{Experimental Materials}

The experiment materials were the same as those used in the previous experiment (Sect. 2.1).

\subsection{Experiment Conditions}

The participants were 20 female university students. This experiment was approved by the Ethics Review Committee of Nara Women's University. The participants were informed of the relevant experimental procedure, and the experiment was conducted with each participant's consent. The test area in the experiment was the forearm segment of the dominant hand, $5 \mathrm{~cm}$ proximal to the wrist joint. The room temperature and environmental humidity were kept at $23{ }^{\circ} \mathrm{C} \pm 0.5{ }^{\circ} \mathrm{C}$ and $50 \pm 2 \% \mathrm{RH}$, respectively, throughout the experiment.

As with the room temperature, the sample cloth was kept at $23{ }^{\circ} \mathrm{C}$. The sample cloths, each differing in water content by $5 \mu$, were prepared in advance and stored in a container with a lid. During the experiment, skin moisture was adjusted by using a wet towel. 


\subsection{Experiment Procedure}

The threshold was evaluated using the staircase method of psychophysical experiments. The participants were asked to close their eyes until the end of the session.

First, the sample cloth with a water content of $0 \mu \mathrm{l}$ was applied. The forearm of the participant was placed in contact with a new sample cloth of $20 \mu \mathrm{l}$ higher water content than once before. Then, the participant stated whether they felt moisture or not. Once moisture was perceived, a cloth $10 \mu$ l lower in water content was attached to the forearm. This was repeated until moisture was no longer perceived. Once a participant stopped perceiving moisture, a sample cloth $5 \mu \mathrm{l}$ higher in water content was applied to the forearm repeatedly, until moisture was perceived. The level of moisture, associated with moisture perception, was then recorded. The duration of contact was $3 \mathrm{~s}$. After the sample cloth was removed from the forearm, the participants reported again on their perception of any moisture. Then, the forearm was placed back on the hot plate to recover skin temperature.

The whole process was repeated four times, and the average value of the four repetitions was taken as the threshold. In order to prevent variation in the water content of the sample cloth, a set of the sample cloths was prepared again, after the evaluation was completed twice.

All of the participants' skin moisture levels were adjusted with a wet towel that stayed on the arm for $5 \mathrm{~min}$. Thereafter, it was confirmed that the skin moisture increased. The same evaluation was performed four times. To maintain skin moisture levels, skin moisture was adjusted for 5 min again, after two evaluations.

\subsection{Results}

Skin moisture content before and after adjustment is shown in Fig. 6. We confirmed that skin moisture content was successfully increased for each participant, following adjustment.

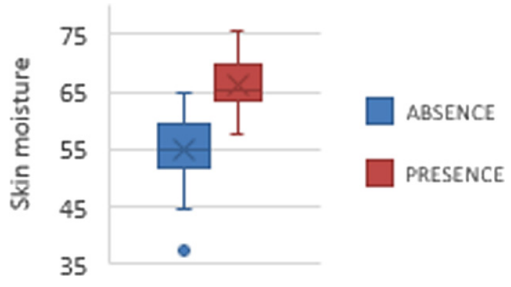

Fig. 6. The skin moisture content levels and after adjustment, in Experiment 2.

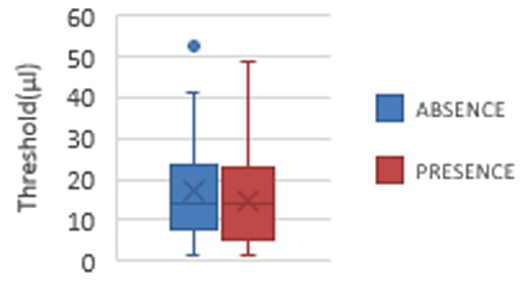

Fig. 7. The threshold of moisture sensation before and after the adjustment of skin moisture levels. The vertical axis represents the threshold value for moisture sensation. 
Figure 7 is a graph summarizing the threshold values before and after the adjustment of skin moisture levels. As shown by the boxplot, the thresholds are almost the same regardless of the presence or absence of a wet towel (before and after the change in the skin moisture). The Wilcoxon signed-rank test revealed no significant difference in skin moisture $(p=0.34)$. It was found that skin moisture did not affect the threshold of the moisture sensation.

\subsection{Discussion}

Experiment 1, in Chapter 2, showed that the participants felt little moisture, when skin moisture was increased; when the level of the skin moisture was high, the threshold of the moisture sensation was presumed to be larger. However, the observed experimental result did not align with this hypothesis. It was found that skin moisture did not affect the threshold of moisture sensation.

We propose that the water remaining on the skin surface, after the removal of the wet towel, affected the observed threshold. When the skin moisture was increased by the wet towel, a small amount of water was left on the skin's surface. There is a possibility that this water decreased the threshold value of the skin. It is also possible that the moisture threshold $(0-30 \mu \mathrm{l})$ was too low to be compared with the water content of the sample cloth $(0,100,300 \mu \mathrm{l})$ in Experiment 1 . We propose that the low water content of the sample cloth led to the diminished skin moisture effect. When an experiment on perceived intensity is performed with a sample cloth having a low level of water content, such as $30 \mu \mathrm{l}$, perceived moisture sensation is expected to be independent of variations in skin moisture. Further research is necessary to characterize the difference in results between the perceived intensity and detection threshold.

\section{Conclusion}

In this paper, we evaluated changes in perceived intensity and the threshold of the moisture sensation in different skin moisture conditions. The results showed that, when skin moisture was increased, participants felt a lower level of moisture. However, the threshold value of moisture sensation was not affected by skin moisture.

In future, we aim to investigate how the discrimination threshold changes with different temperatures and skin moisture conditions. Additionally, we will study how moisture sensation differs across body parts, such as how thresholds differ between the arm and the back.

\section{References}

1. Slater, K.: Human Comfort. Springfield, Ill. C.C. Thomas. USA (1985)

2. Koshiba, T., Tamura, T.: Factors governing the wet sensation of human skin. Jpn. Res. Assoc. Text. End-Uses 36(1), 19-124 (1995)

3. Tamura, T.: A review of studies on regional differences of thermal and humidity sensitivity on human skin surface. Jpn. Soc. Sensory Eval. 11(2), 81-88 (2007) 
4. Shibahara, M., Sato, K.: Illusion of wetness by dynamic touch. IEEE Trans. Haptics 12(4), 533-541 (2019)

5. Shibahara, M., Sato, K.: Illusion of moisture sensation of cloth by thermal control. Jpn. Res. Assoc. Text. End-Uses 56(12), 951-958 (2015). (in Japanese)

6. Carifio, J., Perla, R.: Resolving the 50-year debate around using and misusing likert scales. Med. Educ. 42, 1150-1152 (2008)

7. Ho, H., Jones, L.: Modeling the thermal responses of the skin surface during hand-object interactions. J. Biomech. Eng. 130, 021005 (2008)

8. Tanaka, Y., Sukigara, S.: Evaluation of "shittori" characteristic for fabrics. J. Text. Eng. 54 (3), 75-81 (2008)

9. Okajima, T., Takeda, Y.: Tactile dryness of building materials. Trans. Architect. Inst. Jpn. 327, 12-19 (1983)

10. Jones, L.A., Ho, H.-N.: Warm or cool, large or small? The challenge of thermal displays. Trans. Haptics 1(1), 53-70 (2008)

11. Green, B.G.: The effect of skin temperature on vibrotactile sensitivity. Percept. Psychophys. 21, 243-248 (1977)

Open Access This chapter is licensed under the terms of the Creative Commons Attribution 4.0 International License (http://creativecommons.org/licenses/by/4.0/), which permits use, sharing, adaptation, distribution and reproduction in any medium or format, as long as you give appropriate credit to the original author(s) and the source, provide a link to the Creative Commons license and indicate if changes were made.

The images or other third party material in this chapter are included in the chapter's Creative Commons license, unless indicated otherwise in a credit line to the material. If material is not included in the chapter's Creative Commons license and your intended use is not permitted by statutory regulation or exceeds the permitted use, you will need to obtain permission directly from the copyright holder.

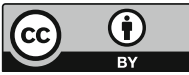

